\title{
Aggregation by very large numbers
}

\section{Computer models of aggregating systems have thrown a useful light on physical processes in the past few years. But too much computer power may make them more intricate than instructive.}

THE neater the model, the more easily it is elaborated, and made complicated. That simple truth, always apparent, has been more evident than ever since computer power became more easily and cheaply available. The model of simple harmonic motion represented by the idealized simple pendulum could, no doubt, be made too complicated for a Cray computer to handle by the wooden elaboration of enough complexity.

Much the same, it seems, is now happening to the simple model of diffusionlimited aggregation first put forward four years ago by T. Witten and L.M. Sander (Phys. Rev. Lett. 47, 1400; 1981). The original idea was simple, that of constructing a model to account for processes such as crystal growth whose rate is determined by the diffusion of aggregating particles through solution towards the growing crystal.

The simple version of the model is that in which aggregation takes place on a twodimensional lattice. Particles are allowed to "diffuse" from the periphery of a piece of the lattice towards a central point by supposing that they move randomly, one lattice step at a time. It is necessary to specify the set of rules that determine when a particle will stick to the growing aggregate; most simply, it can be required to stay where it is whenever it reaches a lattice-site that is immediately adjacent to one already occupied.

Several important demonstrations have been possible with this simple, almost crude, model. Not least, for example, it turns out that aggregates constructed in this way are fractal structures; the numbers of particles they contain increase monotonically with increasing radius, but by some power of the radius which is less than 2.0 , the value expected for a structure filling all the dimensions available in a plane. The value of the exponent in the power law with radius is now the familiar fractional dimension. The fact that just such behaviour is observed when dendritic solid structures grow from, say, solution, or when ice crystals form from the vapour, justifies the wide use made of the model and its elaborations in the past few years.

One side benefit of this simple model is that it lends itself easily to computer calculations. Tell the machine the rules, and it will replicate them indefinitely. So it has come about that a small army of ingenious people has been trying variants of the Witten-Sanders model. More than two dimensions? No problem, except for the cost of computer time. What is the rate at which smaller aggregates will stick together to form larger structures? Again there is no problem once the rules are specified. The fact that the WittenSanders model also serves to account for phenomena other than diffusion-limited aggregation, percolation for example, has made it even more popular.

Naturally, there has been a great deal of interest in attempts to make the model realistic. Obviously, in a model growing by random aggregation, there is a possibility that loops will form between one part of the structure and another, thus excluding a large part of the accessible space from occupation. Are these physically realistic conditions, or should they be excluded as rare configurations with extraordinary implications?

Then the simplest set of rules, requiring that particles should stick to an aggregate whenever they reach a neighbouring site, makes no allowance for the likelihood that different sites might be occupied with different affinity. That is why some investigators have set out to calculate the probability that various sites will be occupied by running the random walk backwards.

More recently, attempts have been made to construct still more realistic models of aggregation by changing the rules, allowing a particle either to stick at the first nearest-neighbour site it reaches or, alternatively, to move to a neighbouring occupiable site, perhaps because such a site may be energetically more favourable. (Two neighbours are better than one.) There are reasons to suppose that the results of such calculations may enjoy a degree of realism (see P. Meakin and $\mathbf{R}$. Jullien, J. Phys. 46, 1543, 1985).

The most recent development is an attempt to construct a kind of equilibrium model of aggregation. The idea (due to $R$. Botet and R. Jullien of the University of Paris-Sud (Phys. Rev. Lett. 55, 1943; 1985 ) is to begin with an aggregate of some kind and arrange that particles may escape from peripheral sites with single nearest neighbours, wander about by random walking (again on a two-dimensional lattice) and then stick to an aggregation site when next they reach it. If the wandering particle should get too far away, say to the circumference of a distant circle, it will be captured and released again at a random point.

Technically, there is no doubt that the aggregating particle is stable. The number of particles is essentially constant; the rules specify that there can never be more than one particle on its travels. So what do the calculations show about the way in which the shapes of aggregates change with the repeated release and recapture of particles at the periphery?

First, for a specified number of particles in the initial aggregate, there is a final state with recognizable characteristics determined only by the number. For example, starting with a cluster of 100 particles allowed to randomize themselves by these formal rules, Botet and Jullien show that the radius of gyration of the end result is roughly eight unit lattice spaces. Attentuated structures (say, a string of 100 particles in a straight line) will finish up as a spindly structure. So too will structures that begin as compact clusters, with no spaces between occupied sites.

The obvious disappointment is that the model, as specified, is not a model for aggregates in equilibrium, but instead a model for an aggregate whose size has been arbitrarily fixed. Put simply, it is a model that presupposes that disaggregation is not an option for the system, or that the energy of the attachment of particles to aggregate is infinite. In the circumstances, especially because there can be no attempt to distinguish between the probability with which different kinds of clusters can occur, which is the same as saying that the energy of all configurations is the same. In other words, the model is a long way from being a model of equilibrium aggregation.

That does not. of course, imply that the calculations are without interest. But it is not surprising that the fractal dimensions of aggregates held constant under these conditions should be less than those of the aggregates formed in the first place by the Witten-Sanders process (1.54 against $1.70)$. Nor is it remarkable that some configurations built from very small aggregates (of, say, eight particles) should occur less frequently than sheer chance dictates. The promised calculation of what happens when larger aggregates split in two, by the severance of a bond chosen at random, may however be more directly relevant to speculation about the way in which aggregates rearrange themselves. The pity is that, for the time being, there are no usable analytical methods of making progress with these problems.

John Maddox 